\title{
Mercadotecnia social digital para promover el interés estudiantil en elecciones de autoridades en la Universidad de Guayaquil-Ecuador.
}

\section{Digital social marketing to promote student interest in elections of authorities at the University of Guayaquil-Ecuador.}

Ing. Luis Adrian Choez Acosta Msc. ${ }^{1}$, Ing. Blakeslees Streisand Suárez Muñoz Msc. ${ }^{2}$, Ing. Alexandra Aracely Navarrete Cornejo. Msc. ${ }^{3}$ \& Ing. Nuvia Lucrecia Morán Sanchez Msc. ${ }^{4}$

Recibido: 14-10-2018 / Revisado: 17-11-2018 /Aceptado: 03-12-2018/ Publicado: 05-01-2019

\begin{abstract}
.
DOI: $\underline{\text { https://doi.org/10.33262/cienciadigital.v3i1.246 }}$

The elections, held in October 2016, were the first in the history of the University of Guayaquil of a universal nature and where students who have passed the first year of studies can participate directly.

This situation was new for the candidates for authorities of the University of Guayaquil, who are accustomed to a more personalized electoral campaign, because the universe of voters was very small.

This article aims to solve this problem, using social networks as strategic tools of EMarketing.

The objective of the present investigation is to determine if the E-Marketing, through the Social Networks and the digital media, would imply to capture votes of the students of the University of Guayaquil in the elections to university authorities.

The methodological approach used was quantitative, the information was obtained through surveys made to students of the different faculties of the University of Guayaquil. It was concluded that the majority of the members of the student community considered that the interaction with the possible candidates for rector and vice chancellors of the University of Guayaquil, through social networks or by some digital means, would positively influence their voting decision. in the elections of university authorities of October 2016.
\end{abstract}

\footnotetext{
${ }^{1}$ Universidad de Guayaquil, Guayaquil, Ecuador, luis_adrian01@yahoo.com

${ }^{2}$ Universidad Agraria, Ingeniería Agrícola, Milagro, Ecuador, nubetransparente@yahoo.com

${ }^{3}$ Universidad Agraria, Ingeniería Agronómica, Milagro, Ecuador, anavarrete@uagraria.edu.ec

${ }^{4}$ Universidad Agraria, Ingeniería Agronómica, Milagro, Ecuador, nsanchez@uagraria.edu.ec
} 
The beneficiaries of this work were the candidates for rector and vice chancellors of the University of Guayaquil and the community in general.

Keywords: E- Marketing, Social Networks, Politics 2.0, University

\section{Resumen.}

Las elecciones, que se realizó en octubre de 2016, fueron las primeras en la historia de la Universidad de Guayaquil de carácter universal y en donde los estudiantes que hayan aprobado el primer año de estudios podrán participar en forma directa.

Esta situación fue nueva para los candidatos a autoridades de la Universidad de Guayaquil, quienes están acostumbrados a una campaña electoral más personalizada, debido a que el universo de electores era muy reducido.

El presente artículo pretende darle solución a dicho problema, utilizando las redes sociales como herramientas estratégicas de E-Marketing.

El objetivo de la presente investigación es determinar si el E-Marketing, a través de las Redes Sociales y los medios de comunicación digital, incidiría en captar votos de los estudiantes de la Universidad de Guayaquil en las elecciones a autoridades universitarias.

El enfoque metodológico empleado fue cuantitativo, la información se obtuvo mediante encuestas realizadas a los estudiantes de las diferentes facultades de la Universidad de Guayaquil. Se pudo concluir que la mayoría de los miembros de la comunidad estudiantil consideraron que la interacción con los posibles candidatos a rector y vicerrectores de la Universidad de Guayaquil, a través de las redes sociales o por algún medio digital, influiría positivamente en su decisión de voto en las elecciones de autoridades universitarias de octubre del 2016.

Los beneficiarios de esta labor fueron los candidatos a rector y vicerrectores de la Universidad de Guayaquil y la comunidad en general.

Palabras claves: E- Marketing, Redes Sociales, Política 2.0, Universidad

\section{Introducción.}

En las elecciones presidenciales del año 2016 en Estados Unidos el corresponsal de la BBC Rory Cellan-Jones declaro que para el equipo de campaña de Trump, las redes sociales Facebook y Twitter fueron medios poderosos para llegar directamente a los electores, aunque los diarios más importantes apoyaron a la candidata demócrata, Hillary Clinton o evitaron respaldar a su rival republicano, Donald Trump. Con esto los periódicos como el resto de los 
medios más significativos se dieron cuenta que su contribución a la campaña de un candidato parece ser mínima cuando se mide con la de Facebook y otras redes sociales.

Según el diario ecuatoriano El Comercio del 15 de abril de 2015 en un estudio que realizo el INEC, Instituto Nacional de Estadística y Censos, en Guayaquil y Quito el 97\% de la población posee una cuenta en estas estructuras que relacionan a individuos con intereses comunes.

El objeto de estudio de este trabajo es la utilización del mercadeo social digital para captar la preferencia de los estudiantes de la Universidad de Guayaquil en elecciones de autoridades universitarias.

La de Ley de Educación Superior en su artículo 55 expresa: "La elección de Rector o Rectora y Vicerrector o Vicerrectora, Vicerrectores o Vicerrectoras de las universidades y escuelas politécnicas se hará por votación universal, directa, secreta y obligatoria de los profesores o las profesoras e investigadores o investigadoras titulares, de los y las estudiantes regulares legalmente matriculados a partir del segundo año de su carrera, y de las y los servidores y trabajadores titulares. No se permitirán delegaciones gremiales."

En estos comicios se aplicó voto ponderado por sectores, como lo indica el art 57 de la citada Ley Orgánica de Educación Superior, LOES: "La votación de las y los estudiantes para la elección de rector o rectora y vicerrector o vicerrectora, vicerrectores o vicerrectoras de las universidades y escuelas politécnicas públicas y privadas, en ejercicio de su autonomía responsable, equivaldrá al porcentaje del $10 \%$ al $25 \%$ del total del personal académico con derecho a voto". Hay que hacer énfasis en que el mayor porcentaje del electorado estudiantil está formado por jóvenes entre 18 y 35 años.

Valiéndonos de lo antes citado y partiendo del problema que tienen los candidatos a una dignidad de autoridad en las Universidades en el Ecuador por captar el interés de los estudiantes se realizó este análisis que promete realizar una mercadotecnia convenientemente económica si se lo contrasta con el alcance y resultado que se puede lograr en la optimización de la capacidad comunicacional de los postulantes con el sector estudiantil.

Se espera utilizar esta clase de mercadeo, debido a su impacto global que goza esta herramienta política ya que fomenta el contacto y la información entre los candidatos a autoridades y el sector estudiantil.

El tema desarrollado en el presente artículo en este establecimiento servirá para que otras instituciones de educación superior puedan reproducir este modelo que da a conocer a sus candidatos.

Según YEPEZ (2010) en la Guía de Elaboración de Proyectos indica que la Investigación de Campo: "Es el estudio sistemático de problemas, en el lugar en que se producen los 
acontecimientos con el propósito de descubrir, explicar sus causas y efectos, entender su naturaleza e implicaciones, establecer los factores que lo motivan y permiten predecir su ocurrencia”. (p. 9)

YEPEZ (2010) indica que la investigación descriptiva interpreta la naturaleza para presentar una interpretación correcta, mientras que Hernández Sampieri (2013) sostiene que describir es lo mismo que medir. En nuestro caso será la caracterización del electorado estudiantil de la Universidad de Guayaquil y sus preferencias con respecto al tipo de redes sociales utilizadas, así como la información de las cualidades que de acuerdo al estudiantado debería tener un candidato autoridad universitaria.

\section{Materiales y Métodos}

Se desea demostrar que si se utiliza el E-Marketing, mediante las redes sociales y los medios de Comunicación digital, en las elecciones para rector y vicerrectores de la Universidad de Guayaquil que se realizó en octubre de 2016, se optimiza la capacidad comunicacional de los candidatos con el sector estudiantil, lo que beneficiará a los postulantes.

El enfoque manejado ha sido cuantitativo, que, mediante la recolección de datos, y basado en el análisis estadístico, permite establecer patrones y probar hipótesis. (Hernández Sampieri, Fernández Collado, \& Baptista Lucio, 2010).

El instrumento de recolección de datos que se utilizó fue la encuesta cerrada con medición de actitudes escala Likert, escalas de intervalos, de afirmación y negación tipo descriptivo explicativo, realizada en la semana del 18 al 22 de julio de 2016, la misma que estuvo compuesta por cinco preguntas dirigida a los estudiantes de la Universidad de Guayaquil. Proporcionándole a los encuestados un tiempo de 10 minutos.

Las edades de los estudiantes que formaron parte de la muestra se encuentran ubicadas principalmente en dos rangos: el primero entre 18 y 23 años, con el $76 \%$ y el segundo entre 24 y 29 años, con el $18 \%$.

La validación de la encuesta se realizó con el software estadístico Statistical Package for the Social Sciences (SPSS). La tabulación de los datos fue ejecutada utilizando la hoja electrónica Microsoft Excel

La población estudiada fueron los estudiantes de la Universidad de Guayaquil, que según el cuadro de los alumnos matriculados obtenido de la página web de dicho centro, a enero del 2016 eran 63.396 estudiantes.

Para obtener la muestra necesaria, para el presente trabajo, se utilizó la fórmula:

$$
\mathrm{n}=\frac{N \sigma^{2} z^{2}}{(N-1) e^{2}+\sigma^{2}+Z^{2}}
$$


$\mathrm{n}=$ tamaño de la muestra

$\mathrm{N}=$ población $\mathrm{e}=$ error maestral

$\mathrm{Z}=$ nivel de confianza

$\sigma=$ desviación estándar

$$
\begin{gathered}
n=\frac{\left(1.96^{2}\right)\left(0.5^{2}\right)(63397)}{0.05^{2}(63396)+\left(1.96^{2}\right)\left(0.5^{2}\right)} \\
n=388
\end{gathered}
$$

En esta labor se identificó: Los medios de comunicación digital más utilizados por los estudiantes, frecuencia con la que los manipulan las mismos, conocimiento de quienes son los candidatos, si es importante que estos postulantes hagan campaña en estas redes contribuyendo a mejorar la comunicación política de los candidatos a autoridades de la Universidad de Guayaquil con el sector estudiantil.

Como principal referente empírico para el presente trabajo de titulación se tomó el estudio denominado: Comunicación electoral universitaria a través de la web 2.0, elaborado por la Dra. Montserrat Vázquez-Gestal, Dra. Ana-Belén Fernández-Souto y el Dr. Jesús PérezSeoane todos docentes de la Universidad de Vigo. En dicho estudio los autores hacen un análisis de las elecciones a rector de la Universidad de Vigo (España) en el año 2010, en donde las tecnologías de información y comunicación tuvieron un papel preponderante durante los comicios.

Se tomó este trabajo de investigación debido a que guarda relación con el presente trabajo de titulación, al analizar el uso de las redes sociales y medios de comunicación digital como herramientas de marketing electoral en elecciones de rector de una universidad, y de cómo esas estrategias de E-marketing impactaron en los jóvenes estudiantes de dicho centro de estudios superiores de España.

Otro trabajo que se consideró fue El Marketing Político a través de Facebook y su incidencia en las elecciones presidenciales del Ecuador en el 2013, en los jóvenes de 18 a 23 años de la Universidad San Francisco de Quito, cuya autora es Andrea Carolina Torres Rodríguez, guardando similitud ya que se considera la influencia de la red social Facebook como herramienta de marketing político.

\section{Resultados}


La presente investigación obtuvo información a través de encuestas realizadas a los estudiantes de la Universidad de Guayaquil, previa la constatación de que se encontraban matriculados como mínimo en tercer semestre de sus respectivas carreras, a quienes se les aplicó el instrumento correspondiente. Esta institución cuenta con extensiones de sus carreras, pero la encuesta se realizó en el campus principal denominada Ciudadela Universitaria Salvador Allende, que es donde se encuentran ubicadas la mayoría de las facultades.

A continuación, los resultados obtenidos de las preguntas de la encuesta:

Tabla 1. Ordenar datos cuantitativos

\begin{tabular}{lc} 
Medios de comunicación digital & utilizados por los estudiantes \\
\hline Alternativa & Frecuencia \\
\hline Facebook & 317 \\
Twitter & 128 \\
Instagram & 149 \\
WhatsApp & 349 \\
YouTube & 143 \\
Correo Institucional & 165 \\
Correo Personal & 263 \\
Otro & 35 \\
\hline
\end{tabular}

Fuente: Encuesta realizada

Tabla 2. Frecuencia de conexión

Frecuencia de conexión a medios de comunicación digitales

\begin{tabular}{lcc}
\hline Alternativa & Frecuencia & Porcentajes \\
\hline De 0 a 3 veces & $\mathbf{1 2 6}$ & $\mathbf{3 3} \%$ \\
De 4 a 6 veces & $\mathbf{1 1 0}$ & $\mathbf{2 8} \%$ \\
7 veces o más & $\mathbf{1 5 2}$ & $\mathbf{3 9} \%$ \\
\hline Total & $\mathbf{3 8 8}$ & $\mathbf{1 0 0} \%$ \\
\hline
\end{tabular}

Fuente: Encuesta realizada

Tabla 3. Conocimiento de los estudiantes 
Conocimiento de los estudiantes sobre los posibles candidatos

\begin{tabular}{lcc}
\hline \multicolumn{1}{c}{ Alternativa } & Frecuencia & Porcentajes \\
\hline SI & $\mathbf{4 5}$ & $\mathbf{1 2} \%$ \\
NO & $\mathbf{3 4 3}$ & $\mathbf{8 8} \%$ \\
\hline TOTAL & $\mathbf{3 8 8}$ & $\mathbf{1 0 0 \%}$ \\
\hline
\end{tabular}

Fuente: Encuesta realizada

Tabla 4. Punto de vista estudiantil

Punto de vista estudiantil sobre el uso del E-Marketing en la campaña electoral Alternativa Frecuencia Porcentajes

\begin{tabular}{lcc}
\hline SI & 328 & $85 \%$ \\
NO & 60 & $15 \%$ \\
\hline TOTAL & 388 & $100 \%$ \\
\hline
\end{tabular}

Fuente: Encuesta realizada

Tabla 5 E- Marketing

Influencia del uso del E-Marketing en la decisión de voto estudiantil

\begin{tabular}{lcc}
\hline \multicolumn{1}{c}{ Alternativa } & Frecuencia & Porcentajes \\
\hline Totalmente de acuerdo & $\mathbf{7 2}$ & $\mathbf{1 9} \%$ \\
De acuerdo & $\mathbf{2 2 6}$ & $\mathbf{5 8} \%$ \\
En desacuerdo & $\mathbf{5 0}$ & $\mathbf{1 3} \%$ \\
Totalmente en desacuerdo & $\mathbf{4 0}$ & $\mathbf{1 0} \%$ \\
\hline TOTAL & $\mathbf{3 8 8}$ & $\mathbf{1 0 0} \%$ \\
\hline
\end{tabular}

Fuente: Encuesta realizada

Se extrae de los datos que los estudiantes al estar inmersos en las redes sociales y los medios de comunicación digital, esperan que los candidatos a autoridades de la Universidad de Guayaquil utilicen los mismos canales para llevar a ellos sus propuestas de campaña y recomiendan a los mismos utilizar los siguientes: Facebook, Instagram, Twitter, Correo electrónico y YouTube, los mismos que están en orden de prioridad.

\section{Discusión}

Los encuestados se mostraron interesados en participar como sufragantes en las elecciones para rector y vicerrectores de la universidad de Guayaquil realizadas en octubre de 2016, $56 \%$. 
Los resultados muestran un sector estudiantil que considera que la interacción con los posibles candidatos a rector y vicerrectores de la Universidad de Guayaquil, a través de las redes sociales o por algún medio digital, influiría positivamente en su decisión de voto en las elecciones de autoridades universitarias de octubre del 2016, el 77\%.

Estos porcentajes hacen notar el apremio de desarrollar estrategias en medios de comunicación digital y en particular en las redes sociales basadas en e-marketing.

Si comparamos el campo de estudio de este artículo", con el referente empírico “Comunicación electoral universitaria a través de la web 2.0", se puede observar que el uso de las redes sociales y los medios de comunicación digital como parte de una estrategia de E-Marketing en una elección de autoridades universitarias, son percibidos por parte de los estudiantes como un eje fundamental de la campaña electoral, debido a que los jóvenes no quieren ser meros receptores de un discurso político sino que quieren espacios para interactuar y debatir las propuestas con los candidatos.

\section{Conclusiones}

- A través de la aplicación de los instrumentos de recolección de datos, de la tabulación y análisis de los mismos, se puede concluir que mayoritariamente los informantes expresaron su desconocimiento sobre el proceso electoral, del mes de octubre de 2016, para elegir rector y vicerrectores de la Universidad de Guayaquil y en particular sobre los posibles candidatos a dichas dignidades.

- Los informantes del presente trabajo de investigación están de acuerdo en la utilización de las Redes Sociales y los medios de comunicación digital, como instrumentos comunicacionales para dar a conocer las propuestas de los candidatos a rector y vicerrectores a la Universidad de Guayaquil al sector estudiantil en las elecciones.

- Los medios de comunicación digital que los estudiantes encuestados recomendaron utilizar, a los futuros candidatos a rector y vicerrectores de la Universidad de Guayaquil, en las elecciones de octubre de 2016 y en las futuras elecciones universitarias que se den en dicha Alma Máter, fueron las Redes Sociales: Facebook, Instagram, Twitter y YouTube. El medio de comunicación digital más utilizado es el correo electrónico personal seguido del correo electrónico institucional.

- La mayoría de los miembros de la comunidad estudiantil, investigados consideran que la interacción con los posibles candidatos a rector y vicerrectores de la Universidad de Guayaquil, a través de las redes sociales o por algún medio digital, intervendría efectivamente en su decisión de voto en las elecciones de autoridades universitarias. 


\section{Recomendaciones}

- Se recomienda hacer un estudio exhaustivo de cada una de estas redes sociales por parte de sus directores de campaña con el fin de producir una guía del buen uso del marketing digital como herramienta estratégica política, para implementar este recurso de manera eficiente y lograr que las propuestas de campaña de cada lista impacten a este importante conglomerado, $25 \%$ de los votos.

- A las autoridades de la Universidad de Guayaquil se les recomienda considerar el alto porcentaje de estudiantes que desconocen sobre el proceso electoral de octubre de 2016, para que realicen campañas informativas sobre las mismas.

- De igual manera se sugiere, dado el alto número de estudiantes que no piensan acudir a sufragar, informar sobre las sanciones a las que pueden ser sometidos por no participar en dichos comicios.

- Para futuros estudios referentes al tema se recomienda ampliar la investigación no solo al sector estudiantil si no también al sector docente y de servicio, $75 \%$ de los sufragantes.

- Se exhorta replicar el presente trabajo de investigación en una institución de educación superior privada, con el objetivo de establecer el impacto que pueda tener una estrategia de Marketing digital en el sector estudiantil, en una elección de autoridades universitarias.

\section{Referencias bibliográficas.}

Calderón, C. (2011). Manual del e-lider. En G. Alarcón y J. Llinares (Coords). Política 2.0: las campañas electorales en la red. Granada: Algón.

Caldevilla Dominguez, D. (2009). Democracia 2.0: La política se introduce en las redes sociales. Recuperado el 5 de 08 de 2016, de Pensar la Publicidad, vol. III, 2, 31-48 http://revistas.ucm.es/index.php/PEPU/article/view/PEPU0909220031A/15218

Celaya, J. (2009). La empresa en la web 2.0: el impacto de las redes sociales y las nuevas formas de comunicación online en la estrategia empresarial. Madrid: Gestión 2000.

Christakis, N. (2010). Conectados: el sorprendendente poder de las redes sociales y cómo nos afectan. México D.F: Taurus.

Florido Miguel. (08 de 01 de 2015). Qué es un KPI en Marketing. Ejemplo de SEO y Social. Obtenido de Marketing and Web: http://www.marketingandweb.es/marketing/quees-un-kpi-en-marketing/

Galán Amador, M. (15 de 12 de 2010). Ética de la investigación. Obtenido de Revista Iberoamericana de Educación: http://rieoei.org/jano/3755GalnnJano.pdf

García Sánchez, M. D. (2008). Manual de Marketing. Madrid: Esic Editorial. 
Hernández Sampieri, R., Fernandez Collado, C., \& Baptista Lucio, M. (2010). Metodología de la Investigación 5ta. Ed. México: McGraw Hill.

Historia de la Universidad de Guayaquil. (s.f.). Obtenido de Universidad de Guayaquil: http://www.ug.edu.ec/nosotros/

Kutchera, J. (2014). E-X-I-T-O: su estrategia de marketing digital en 5 pasos. México: Grupo Editorial Patria.

Ley Orgánica de Educación Superior del Ecuador. (12 de 10 de 2010).

Ortiz Rabadán, R. (29 de 04 de 2015). Descubre Marketing. Obtenido de Marketing Político: La importancia de la estrategia en las campañas electorales:http://descubremarketing.com/marketing-politico-campanas-electorales/

Rubira, F. (24 de 06 de 2013). La confidencial digital. Obtenido de ¿Qué es instagram y para qué sirve?: http://www.elconfidencialdigital.com/opinion/tribuna_libre/Instagramsirve_0_2076992284.html

Sanabria, C. (12 de 02 de 2015). Universidad Autónoma de México. Obtenido de Social Media Acatlán: http://blogs.acatlan.unam.mx/smacatlan/2015/02/12/marketingpolitico/

Segovia, P. (12 de 08 de 2011). El Marketing Digital es mucho más que las redes sociales. Obtenido de Blog de Pablo Segovia: http://www.pablosegovia.cl/content/view/1349632/El-marketing-digital-es-muchomas- que-las-redes-sociales.html

Suárez, M. L. (16 de 01 de 2016). Población estudiantil. Obtenido de Universidad de Guayaquil: http://www.ug.edu.ec/poblacion-estudiantil/

Torres Rodriguez, A. (15 de 12 de 2012). Universidad San Francisco de Quito. Obtenido de Repositorio Digital: http://repositorio.usfq.edu.ec/handle/23000/1760

Tuñes, M., \& Sixto, J. (2011). Redes Sociales, política y Compromiso 2.0: La comunicación de los diputados españoles en Facebook. Recuperado el 12 de 08 de 2016, de Revista Latina de Comunicación $\quad$ Social, http://www.revistalatinacs.org/11/art/930_Santiago/RLCS_art931.pdf

Vásquez-Gestal, M., Fernández-Souto, A.-B., \& Pérez-Seoane, J. (2012). Comunicación electoral universitaria a través de web 2.0. Obtenido de Revista Latina de Comunicación Social, 67: http://www.revistalatinacs.org/067/art/961_Vigo/RLCS_paper961.pdf

Weber, L. (2010). El marketing en las redes sociales 2da. ed. Mexico D.F.: McGraw-Hill. 


\section{Para citar el artículo indexado.}

Choez L., Suárez B., Navarrete A. \& Morán N. (2019). Mercadotecnia social digital para promover el interés estudiantil en elecciones de autoridades en la Universidad de GuayaquilEcuador. Revista electrónica Ciencia Digital 3(1), 8-18. Recuperado desde:

http://cienciadigital.org/revistacienciadigital2/index.php/CienciaDigital/article/view/246/54

$\underline{9}$

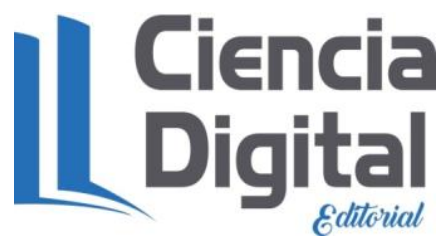

El artículo que se publica es de exclusiva responsabilidad de los autores y no necesariamente reflejan el pensamiento de la Revista Ciencia Digital.

El articulo queda en propiedad de la revista y, por tanto, su publicación parcial y/o total en otro medio tiene que ser autorizado por el director de la Revista Ciencia Digital.
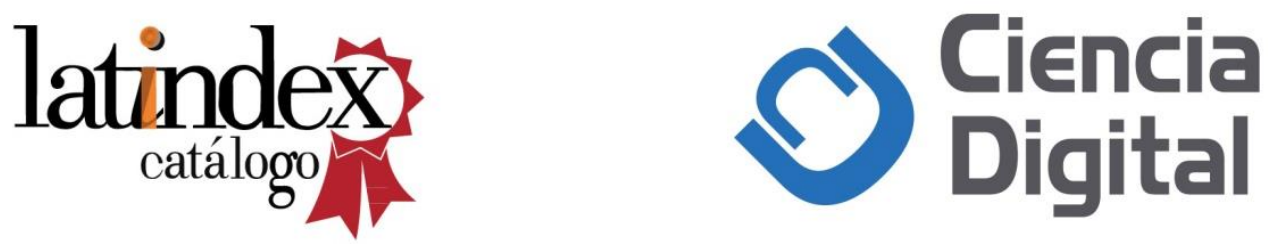